\title{
Precauciones en la Cirugia Abdomino-Pélvica de la mujer
}

Diagnóstico de precision con la pelviscopia

\author{
Por el doctor RODLlfo CAMERO \\ Trabajo presentado a f-imer C nares, Nacional de Cirujanos
}

Desde hace algunos años. la preocupación por cuidar la maycr If́tegridad en los órganos genitales femeninos, ha ido creciendo.

La Cirugía, ante la necesidać de coriservar la función. perfecciona las técnicas, y la Fisiología como la Endocrinología las orienta, apartando a la mujer cada vez más de la esterilidad.

Para ordenar mejor este estudio. lo dividiremos en cuatro secciones. asi:

I-Apendicitis y quistes anexiales. II-Cirugía de los ovarios. III-Cirugía de las trompas. IV-Cirugía de la matriz.

Al final se hará una descripción de la técnica empleada por el doctor Decker, con el aparato de su inverción, el CULDOSCOPE, para diagnósticos de precisión en la pelvis.

I-Apendicitis y quistes anexiales. Es muy frecuente en nuestro medio. la inclinación del cirujano hacia las pequeñas incisiones para apendicectomia en la mujer. En otras ocasiones, en su condescendencia a las peticiones de ella para que proceda en
Esta forma, con fines estéticos. Es asi como casi todos hemos practica. do o presenciado intervenciones de esta indole, por incisiones casi imperceptibles. Pcco tiempo después se encuentra la verdadera lesión, o la ccexistente con el cuadro clínico pr: mitivo, en los anexos.

Los hechos demuestran este cấn en todas las edades, pero es de un.t frecuencia insospechada en las niñas Y mujeres jóvenes. Comoquiera que se trata de lesiones que comprometen esenciales funciones de la mujer. debemos rectificar costumbres reñidas con la técnica y que dejan ui saldc bastante apreciable de secue. las que no se reparan con facilidad.

Debemos considerar una de las causas de error en el diagnóstico: EL EXAMEN INCOMPLETO. Sin tacto vaginal en las multíparas o rectal en las nulíparas, el cirujano está expuesto a tener que reoperar, poco tiempo después, en condiciones desventajosas, o lo que es peor, otro descubrirá el error. Pero como este exa. men no da mucha luz en las obesas, en las pusilánimes, en las solteras o niñas que siempre lo rechazan. así 
como en los casos agudos, se debe recurrir al tacto bajo anestesia. Considerando que $l_{a}$ apendicitis es evidente y que se impone la operación, el examen se simplifica practicándolo momentos antes del acto quirúrgico. cuando la paciente, debidamente premedicada y anestesiada, presenta buen relajamiento de las paredes. Es práctico que el cirujano haga este examen antes de bañarse para la intervención.

Es muy dificil, con una incisión pequeña y especialmente del tipo Mac Burney, operàr una verdadera apendicitis y dejar en buenas condiciones los órganos pelvianos, reparados de las lesiones que más comúnmente se localizan en ellos. Estas son en su mayoría, quistes, hematomas, fibromas pediculados $\mathrm{y}$ adherencias.
Por la brevedad del tiempo asignado a esta exposicion apenas enumeraremos algunos procedimientos.

La celiotomía mediana, sería demasiado cruenta y es bien sabicio que en muchas apendicitis, esta vía no facilita la intervención.

El Phanenstiel que reúne grandes condiciones anatómicas, de estética y que asegura nuevamente la solidez de la pared abdominal, puede presentar también dificultades en las localizaciones altas o retrógradas del apéndice.

La incision de Mac Burney debe reservarse para casos muy especiales y cuando se haya verificado el examen ya citado, sin que esto pue. da dejar al cirujano en completa tranquilidad en relación con los órganos pelvianos.

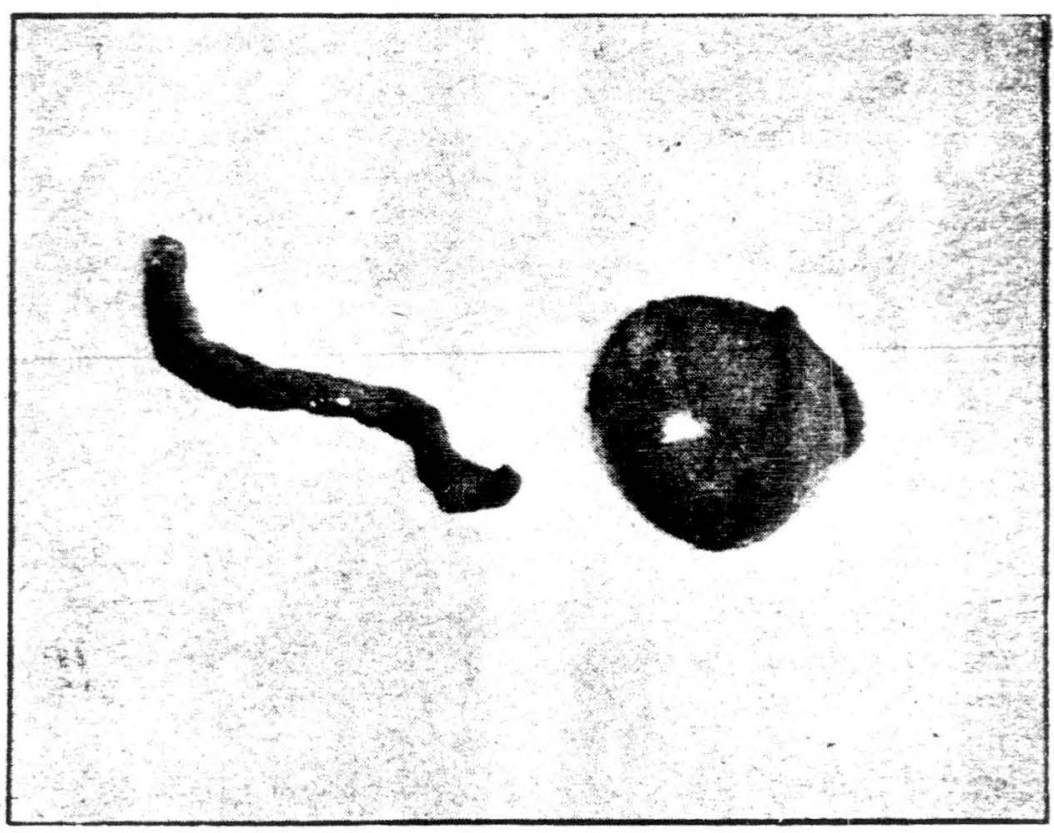

FIGURA 1

Apéndice $\mathrm{y}$ quiste del mesosalpinx 
La incisión de Jalaguier prescnta las mayores comodidades parł cua!quier clase de apendicectomias $\mathbf{y}$ permite explorar e intervenir casi todas las lesiones que hemos anotado en la matriz y en los anexos. La facilidad de ampliarla en cualquiera de sus extremìdades sin comprome. ter demasiado la solidez de la pared abciominal, permite usarla casi rutinariamente. Siendo el anexo izquierdo accesible por esta via, la técnice deja menos qué desear.

Con incisión de Jalaguier fue operada la enferma a quien se le extrajo el apéndice y el quiste que muestra la Fig. $N^{0} 1$, como un ejemplo de los numerosos casos que a diario se presentan.

E1 resultado del examen anatomo. patológico del apéndice, dice asi:

«Conclusión: Trátase de una aper. dicitis crónica, con atrofia de la pa red, dilatación de la cavidad y retención estercoral" (Fdo. M. Mariño Z. El quiste estaba localizado en el me sosalpinx y doña Cecilia Hernández de Paredes da el siguient $\_$resultado de su examen: Densidad 1.012; Glucosa 60 mlgr. \% (Técnica de FolinWu); Urea $41 \mathrm{mlgr}$. \% (Técnica de Karr); Citológico: Linfocitos 100\% No se observan otras células.

No dejamos de insistir en que el examen bajo anestesia decidirá la técnica de la intervención.

II-Cirugía de los ovarios. Como sólo nos ocuparemos de los tumores líquidos, se debe tener el cuidado, especialmente en las -mujeres jóvenes, de conservar lo que quede de sustancia orgánica tratándola comc el resto de una resección cuneiforme. Es muy satisfactorio ver en estos ca- sos, así sean bilaterales, que la mens . truación continúa y poder comprobar la ovulación por medio de la temperátura basal o de la biopsia.

Los quistes de las trompas se de. ben disecar, peritonizando la brecha nuevamente.

III-Cirugía de las trompas. La comentamos únicamente en lo quz hace relación a la mujer antes de la menopausia y con fines de corregir los obstáculos a la concepción.

Por la irrigación especial que tienen, como lo podemos observar en la figura $N^{\circ} 2$.

Se ve claramente, que en cualquier parte que se seccione, le queda irrigación. Con algunos procedimientos, como el del doctor Axel Westmann, se puede extirpar una porción de la trompa y restablecer la comunicación entre los dos cabos, o entre la extremidad distal y el cuerno de la matriz cuando se trata de incluirla, colocando en la luz de estas porciones una barra de oleato de colesterol sobre la cual se hará la sutura. circular de los cabos.

Como el punto de fusión de esta sustancia es de 39 a 40 grados, ai sexto u octavo día de la intervención se provocará una elevación de la temperatura con la aplicación a la paciente de una vacuna, lo que hará disolver el oleato de colesterol que pasará a la cavidad abdominal o a la matriz, sin inconveniente alguno. Este método ha dado algunos éxitos confirmados por embarazos posteriores.

Otra técnica consiste en pasar una sonda ureteral a través de la porción distal de la trompa y llevarla por el cuerno uterino hasta el cuello y la 


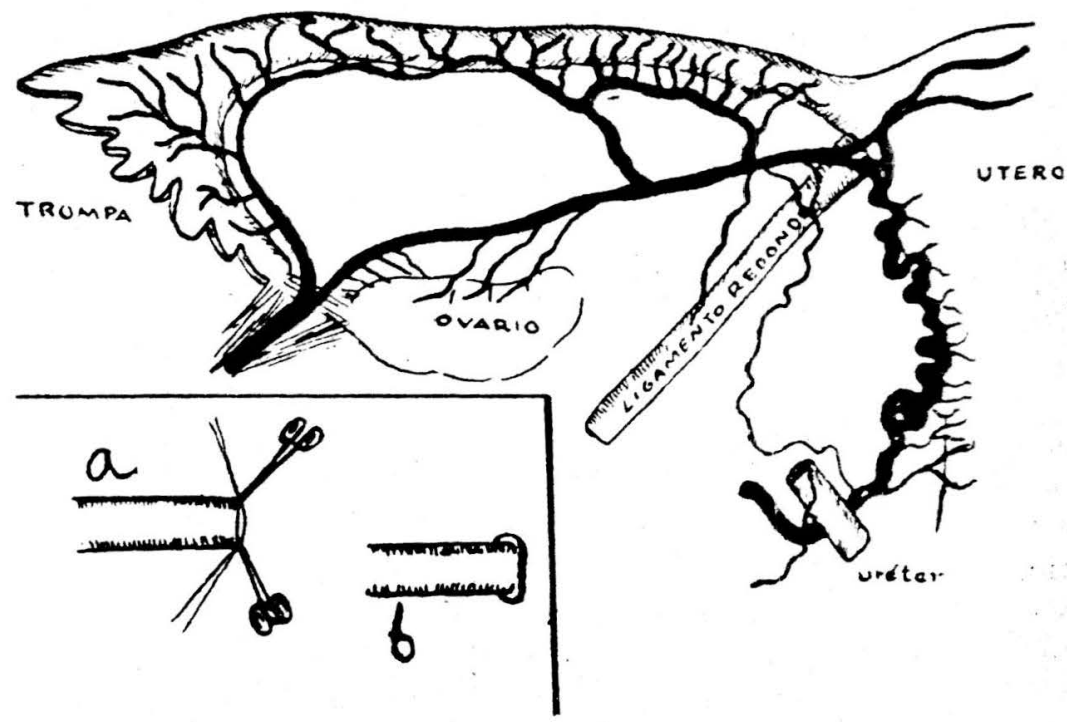

Figura 2

Distribución circulatoria de la trompa

(Dibujo del Dr. Arturo Marroquin)

vagina, pudiendo ser el procedimiento bilateral, y las sondas se fijarán a la salida de la vulva. Previamente se abre el cabo de la trompa que se va a insertar en el cuerno de la matriz y se preparan los puntos con que se ha de fijar. Al cabo de seis dias se retiran las sondas cuida dosamente $\mathbf{y}$ se prueba la permeabilidad, tanto en esta técnica como en la anterior por medio de persuflaciones simples sucesivas. Esta última técnica fue practicada el año pasado por los doctores Alfredo Jakob y Guillermo Di Paola en la ciudad de Buenos Aires. Ignoro los resultados, pero por la literatura general el resultado no es siempre halagüeño.

El doctor Randolph Gepfert de Nueva York, está practicando una técnica muy simple, cuyos detalles se encuentran en la esquina de la figura $\mathrm{N} 2^{\circ}$, y consiste en ligar los bordes inferior y superior de la extremidad proximal, con fines hemostáticos como lo muestran las pinzas del grabado. No se hace ninguna otra ligadura y se prescinde del cabo distal o se extirpa si las condicianes patológicas lo exigen. Así, el cabo proximal quedará abierto, la mucosa prolifera cubriendo los bordes al estilo de la Figura b, y queda en condiciones de recoger el óvulo maduro. El doctor Gepfert rolata varios casos de embarazos posteriores a esta intervención. También se debe buscar la permeabilidad con las persuflaciones.

IV-Cirugía de la matriz. En cuànto a los fibromas, de todos es sabido, que se debe intentar enuclearlos hásta donde sea posible, respetando la 'integridad del órgano. En caso de que esto no sea posible, viene la intervención mutilante y que se debe 
definir por HISTERECTOMIA TOTAL O SUB-TOTAL.

Te Linde, de Baltimore, en su obra "Operative Gynecology", manifiesta la posibilidad de elegir una $u$ otra, descontando de antemano las que obedezcan a lesión maligna comprobada, en cuyo caso es bien sabido, se hará radical.

La incidencia del carcinoma en el cuello restante era considerada por este autor, hace algunos años, como de poca significación, ya que en su práctica privada hasta 1945 , sólo contaba dos casos en total. Seguramente en la próxima edición de su obra modificará este concepto, pues ya en junio de este año, durante la Centésima Reunión Anual de la Asociación Médica Americana, en Atlantic City, hizo alusión al aumento del porcentaje.

También el doctor Meigs, de Boston, durante la reunión en que la Sociedad Americana de Ginecología celebraba sus bodas de diamante en la ciudad de Nueva York, en mayo de este año, hizo alusión al aumento de estas incidencias, en conferencia dictada en el Memorial Hospital de la misma ciudad. En este hospital, dedicado exclusivamente a cirugía e investigación del cáncer, el doctor Brunckswig, cirujano de fama, no practica desde hace muchos años la histerectomía sub-total.

En la Argentina y Brasil, comparten la opinión del peligro que representa para el futuro de la mujer, el muñón cervical.

En Berlín, el doctor Nordmeyer y en Estocolmo Axel Westmann, Profesores de Ginecología, así como el doctor Kottmeier, Director de Insti- tuto de Radium en la capital Sueca, consideran un error $y$ una temeridad, el cuello restante.

En Colombia y por el corto tiempo transcurrido entre mi regreso y la fecha de este Congreso, sólo pude captar las opiniones y algunos datos estadisticos de Bogotá. Esta circunstancia me ha privado de contar con las valiosas opiniones de colegas en las otras ciudades del país, así como de otros datos estadísticos. El doctor Daniel Brigard ha insistido desde hace muchos años. en el Instituto de Radium y en la cátedra de Ginecología sobre la inutilidad y el peligro de la histerectomía sub-total. Con sus compañeros de directiva en el Instituto. denominaron gráficamente «cáncer restante del cuello restante:, a la lesión que aparecía algún tiempo después de la operación mencionada y que no era otra cosa que la extirpación parcial de una lesión maligna previamente establecida, pero mal diagnosticada.

El doctor Roberto Restrepo, con su gran experiencia en Radiumterapia, no sólo manifiesta su oposición a la histerectomia sub-total, sino que afirma alarmado. lo incurable o por lo menos hostil al tratamiento de la lesión que nos ocupa

El doctor Cesar Pantoja, durante los siete años en que estuvo como Director del Instituto Nacional de Radium, confirmó su opinión sobre el riesgo que implica el muñón cervical. Pudo apreciar de cerca la dificultad en el tratamiento del cáncer restante, pues no hay cómo fijar los colpostatos y si se trata de operación, también es de difícil ejecución.

El doctor Guillermo López, agregado a la cátedra de Ginecología, 
sólo practica la sub-total sen caso de endometriosis externa, en que la reacción pericervical puede comprometer demasiado el recto y correría el risgo de abririo.

A pesar de todos esos conceptos, no se ha insistido lo suficiente y por eso registramos en los últimos cinco años en algunos hospitales y clínicas de Bogotá el siguiente número de histerectomias sub-totales:

Hospital de la Samaritana: 208.

Hospital de San José: 310.

Hospital de San Juan de Dios: Clínica de Marly:

Clínica del doctor Camero: 11.

\section{PELVISCOPIA}

Renunciamos al nombre de Culdoscopia con que generalmente se denomina el uso del aparato del doctor Decker, o CULDOSCOPE, para el examen de la pelvis.

$\mathrm{El}$ nombre de este aparato lo escogió su inventor, con base en la región que atraviesa y que los franceses llaman «cul-de-sac. Pero como vemos claramente que se usa para explorar los órganos contenidos en la pelvis, a saber: útero, ovarios, trompas, y además es posible observar el apéndice, intestino y uréteres, nada más apropiado que llamar pelviscopia a esta exploración a la manera de los otros estudios que se hacen en vejiga, estómago, etc. Además la palabra Cuidoscopia no tiene aplicación si se tiene en cuenta su raj́z griega. En tal caso el fenómeno de perforar el fondo de saco podría llamarse Culdotomía.

Hasta hace algunos años se venía explorando la región de la pelvis con el Peritoneoscopio, pero las adheren. cias, distensiones intestinales, curvatura del sacro y especialmente la posición de decúbito dorsal, dificultaba detallar los anexos.

Vino entonces el aparato del doctor Decker, con el cual se ha conseguido una magnífica exploración, y actualmente en los Estados Unidos se está ensayando en todos los hospitales con resultados altamente satisfactorios, especialmente en el Knickerbocker Hospital de Nueva York, donde trabaja su inventor.

Descripción del aparato y accesorios.-En la Fig. 3 está distribuido el equipo completo para la pelviscopia y su utilización es como sigue:

En primer término está el frantoluz, que facilita sobremanera la localización del sitio de anestesia y de punción. En seguida está la pinza de curación uterina para hacer la desinfección completa de la vagina. luégo las valvas para levantar el tabique recto-vaginal y las pinzas para fijar el labio posterior del cuello. A continuación está la jeringa de $10 \mathrm{c}$. c., con aguja larga de bisel corto, y después de ésta el trócar que introducido en el tubo siguiente y fijado por la palanca, servirá para perforar el fórnix posterior. Por último está el tallo luminoso con su instalación eléctrica conectada al reóstato y al transformador correspondiente.

Técnica.-Preparación de la enferma como para cualquier intervención pelviana, sin olvidar que sirve de mucho el que la vejiga esté vacía. Luégo se coloca la mesa como para un Trendelemburg, en una inclinación como la que muestra la Fig. No 4 . La enferma se coloca en 


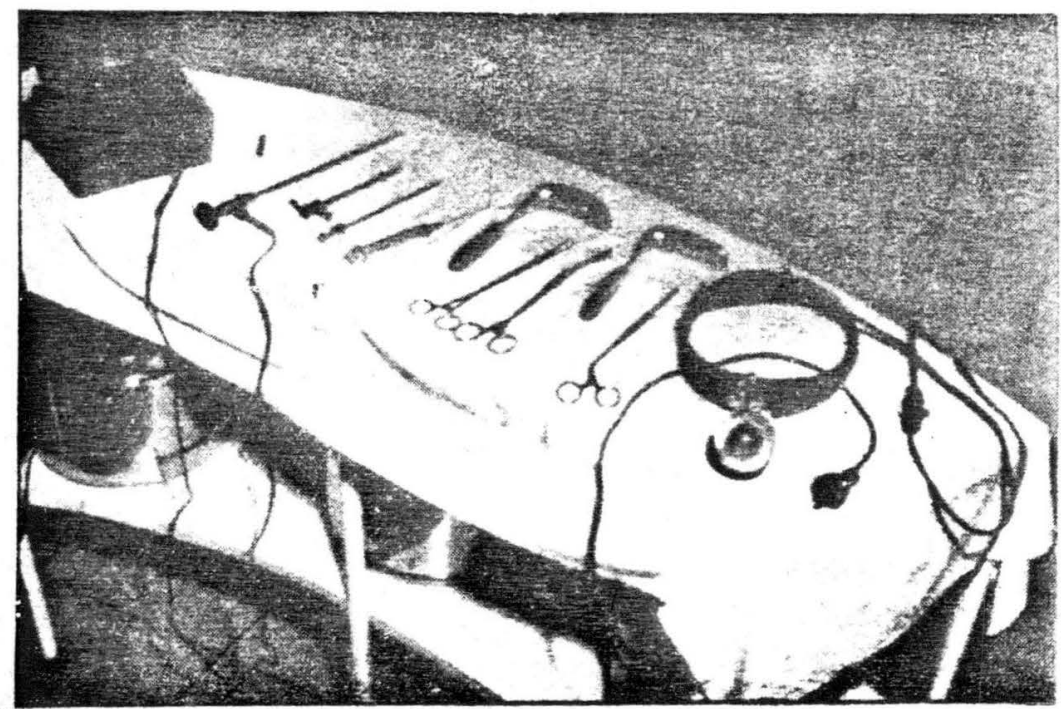

FIGLRA 3

Distribución del equipo para Pelviscopia

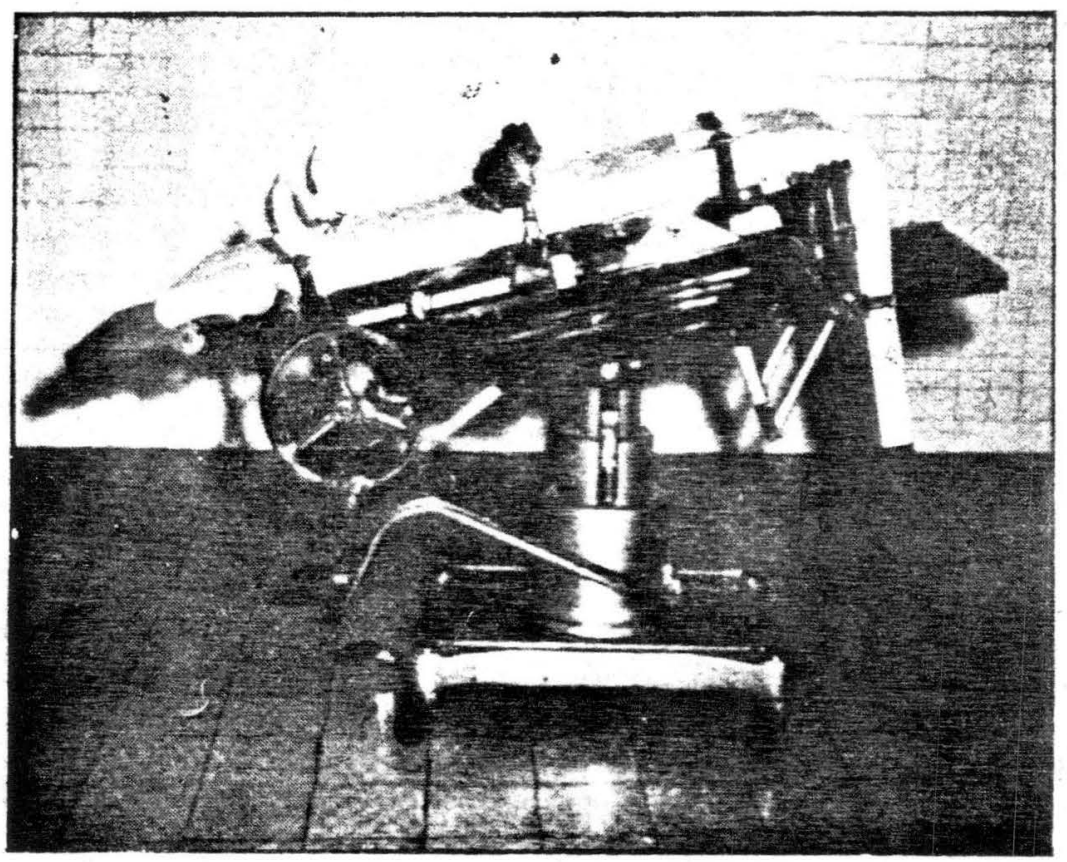

Flalra 4

Posición de la mesa para colocacion de la enferma 
posición genupectoral y si la mesa no tiene el aditamento que muestra la figura para colocar las rodillas, se le dará menos inclinación y la enferma quedará colocada sobre el segundo cuerpo de la mesa. Es indispensable que la pared abdominal quede libre, a fin de que la matriz tenga espacio para desalojarse hacia adelante. En seguida se colocan los campos estelirizados, se pone una valva contra la pared vaginal posterior, se tracciona hacia arriba y se repite la desinfección a la vista. Con la ayuda del fronto-luz se localiza perfectamente el borde posterior del cuello, se fija con una pinza y se tracciona suavemente. Retirado un poco la valva, el fondo de saco posterior sigue al cuello, los ligamentos uterosacros hacen relieve en forma de $\mathrm{V}$ y entre ellos se hunde la mucosa determinando una foseta, como lo muestra la Fig. 5. Traccionando e introduciendo el cuello alternativamente, varias veces, se observan ciertos pliegues especiales de la mucosa que permiten apreciar que no está adherida ni a la matriz ni al recto. Esta cúpula es anestesiada en seguida con dos a tres centímetros cúbicos de cualquier anestésico local y unos minutos después se introduce el trócar suavemente y con firmeza, hasta el reborde saliente del tallo conductor.

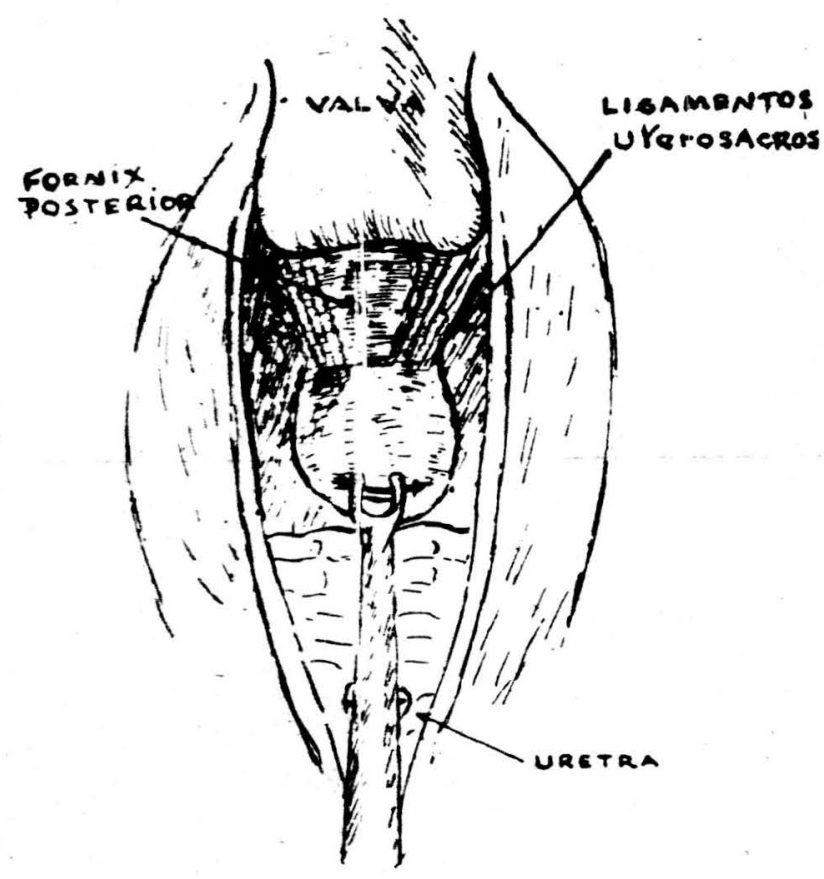

FIGURA 5

Determinación del sitio de punción, por extensión de los ligamentos útero-sacros

(Dibujo del Dr. Arturo Marroquín) 
Luégo se retira el trócar aflojando la palanca que lo asegura y al retirarlo se apreciará un sonido especial como de succión, que caracteriza la entrada del aire a la pelvis. En seguida se coloca el tallo luminoso ya conectado, teniendo en cuenta el sumergirlo en agua templada para que el calor del cuerpo no empañe el espejo en el interior de la pelvis. Se retira el fronto-luz al operador $y$ como toda sala de endoscopia debe ser oscura, se va controlando directamente la parte óptica al mismo tiempo que el aparato entra al Douglas. Orientado según la Fig. No 6 (TeLinde) se hace la exploración a uno $\mathrm{y}$ otro lado, subiendo o bajando el tallo del espejo. Traccionando el cuello suavemente se ayuda a la exploración, lo mismo que levantando la pared abdominal contra la región lumbar, lo que hará un ayudante o enformera. Con el aparato vienan unas cánulas de tornillo, en tres dimensiones diferentes, para adaptar al cuello de la matriz. Cuando se las quiere usar. se articulan a un tallo husco que tiene en sus extremidades el sistema Beckton y quedan listas para conectar con una jeringa o con un Insuflador. De esta manera se puede inyectar líquido de prueba $u$ gas, cuyo obstáculo o paso a través

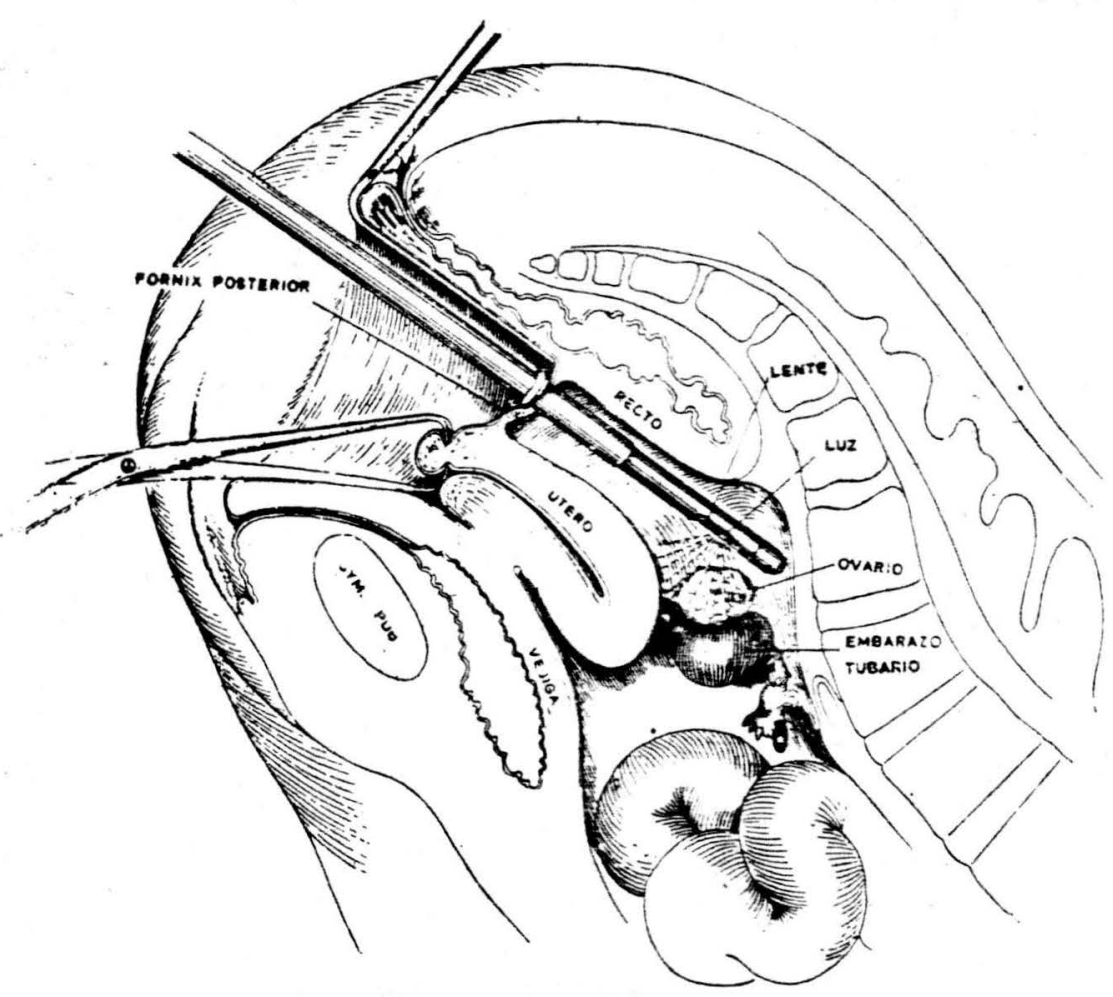

FIGURA 6

Corte sagital que muestra la posicion del aparato y de los órganos pelvianos (American Journal of Obst. and Gyn.) 


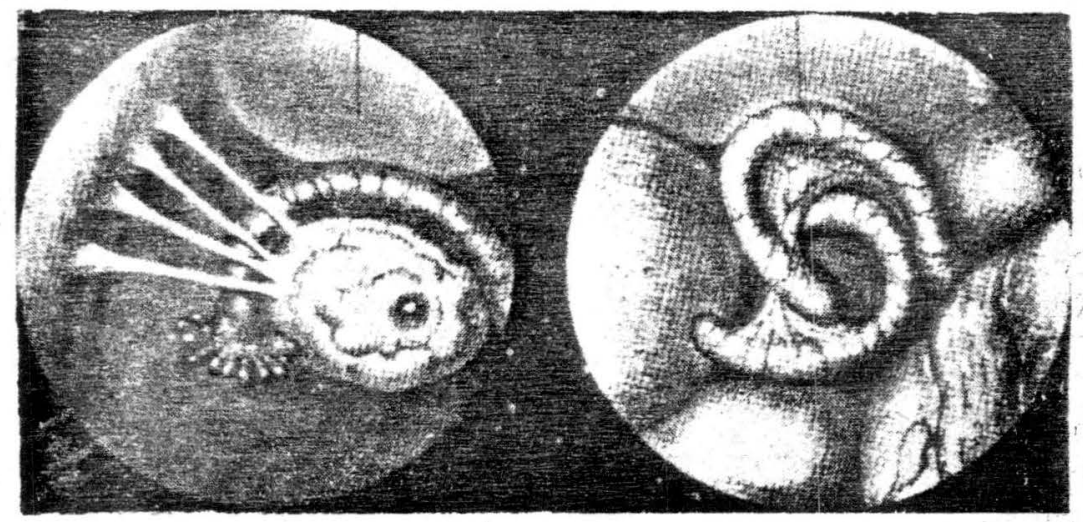

FIGURA 7

Hallazgos a la Pelviscopia. Izquierda: ovario, trompa y adherencias.

Derecha: apéndice y asas intestinales

de la trompa, se comprobará a la pelviscopia. También se emplea para inyectar medios de contraste en la Histerosalpingografia.

Terminada la exploración se retira la parte óptica, dejando el tubo conductor rasta que la enferma sea acostada completamente sobre la mesa en la misma posición genupectoral; con esto sale todo el aire de la cavidad abdominal. y se puede retirar el tubo. No es necesario cerrar la brecha del fondo de saco y es conveniente dejar la $\in$ nferma acostada por 24 horas, en caso de que no resulte ninguna indicación quirúrgica inmediata.

En próximos estudios sobre la misma técnica daremos a conocer sus indicaciones, contraindicaciones y demás comentarios que de su uso se desprenden.

Tan sólo diremos que es muy útil para los diagnósticos de precisión en embarazos ectópicos, quistes anexia- les, endometrosis externa, apendicitis, adherencias, etc. $\mathrm{Y}$ que tiene contraindicaciones como la presencia de una masa fija en el Douglas, la descompensación cardíaca y la vaginitis.

Entre los accidentes que pueden presentarse, está la herida de la cara posterior de la matriz o la perforación del recto. La primera es de poas consecuencias y sobre la segunda, el doctor Decker anota que en su caso particular no suturó la brecha y tampoco se presentaran complicaciones.

REFERENCIAS Ý BIBLIOGRAFIA

1-Archivo de Historias. Clínica del doctor Camero. Bogotá. 1949.

2-Demostraciones quirúrgicas de los doctores Juan C. Ahumada, Alfredo Jakob y Guillermo Di Paola, en la ciudad de Buenos Aires. 1950. 
3-Conferencia del doctor Axel Westmann en el Hospital Karolinska de Estocolmo. 1950.

4-Conferencias del doctor Hans Ludwig Kottmeier en el Radiumhemmet de Estocolmo. 1950.

5-Conferencia del doctor Kurt Nordmeyer en el Rudolf Virchow Krankenhaus de Berlin. 1950.

6-Demostraciones quirúrgicas del doctor Heinrich Martius en la Frauenklinik de Gottingen, Alemania. 1950.

7-Conferencia y demostraciones quirúrgicas del doctor G. Randolph Gepfert en el New York Hospital. 1951.
8-Demostraciones quirúrgicas del doctor Brunsckwig en el Memorial Hospital de Nueva York. 1951.

9-Prácticas del doctor Decker en el Knicherbocker Hospital de Nueva York. 1951.

10-Prácticas del doctor Buxton en el Presbyterian Medical Center de Nueva York. 1951.

11-Te Linde. Operative Gynecology. 1946.

12- Te Linde. American Journal of Obstetrics and Gynecology 1948.

13-Joe V. Meigs. $75^{\circ}$ aniversario de la Sociedad Americana de Ginecología. 1951.

\section{PARA PROPAGANDA EN ESTA REVISTA}

entenderse con el Administrador:

\section{JUAN N. BAQUERO}

en su nueva dirección:

Calle 16, No 7-91 (segundo piso) Oficina $N^{\circ} 4$.

Teléfono 25-641.

Apartado número 276.

BOGOTA - COLOMBIA 